\title{
Insights on Significant Implication on Research Approach for Enhancing 5G Network System
}

\author{
Vani B P $\mathbf{1}^{1}$ R Sundaraguru ${ }^{2}$ \\ ${ }^{1,2}$ Department of Electronics \& Communication Engineering, \\ Sir M. Visvesvaraya Institute of Technology, Bengaluru, India
}

\begin{tabular}{l}
\hline \hline Article Info \\
\hline Article history: \\
Received Apr 4, 2019 \\
Revised Apr 30, 2019 \\
Accepted Aug 29, 2019 \\
\hline
\end{tabular}

\section{Keywords:}

$4 \mathrm{G}$,

$5 \mathrm{G}$,

Resource Allocation, Energy Consumption, Spectral Efficiency, Cognitive Radio Network.

\begin{abstract}
With the exponential growth of mobile users, there is a massive growth of data as well as novel services to support such data management. However, the existing 4G network is absolutely not meant for catering up such higher demands of bandwidth utilization as well as servicing massive users with similar Quality of service. Such problems are claimed to be effectively addressed by the adoption of $5 \mathrm{G}$ networking system. Although the characteristics of $5 \mathrm{G}$ networking are theoretically sound, still it is under the roof of the research. Therefore, this paper presents a discussion about the conventional approach as well as an approach using cognitive radio network towards addressing the frequently identified problems of energy, resource allocation, and spectral efficiency. The study collects the existing, recent researches in the domain of $5 \mathrm{G}$ communications from various publications. Different from existing review work, the paper also contributes towards identifying the core research findings as well as a significant research gap towards improving the communication in the $5 \mathrm{G}$ network system.
\end{abstract}

Copyright ( ( 2019Institute of Advanced Engineering and Science. All rights reserved.

\section{Corresponding Author:}

Vani B P,

Research Scholar, Department of Electronics \& Communication Engg.

Sir M. Visvesvaraya Institute of Technology,Bengaluru, India

Email: vani.smvit@gmail.com

\section{INTRODUCTION}

At present, the usage of the fourth generation or $4 \mathrm{G}$ network is prevalent everywhere globally [1]. However, there are increasing demands of maximized mobile broadband in order to cater up the dynamic application and services e.g., interactive media, $4 \mathrm{~K}$ video streaming, real-time collaboration, remote working environment, virtual reality, etc. However, with the exponential rise of mobile users as well as application the inefficiency of the $4 \mathrm{G}$ network has already surfaced in commercial markets. This leads to hope of fifth generation (5G) network [2], which is claimed to overcome the limitation of 4G. It is claimed that usage, as well as the adoption of the $5 \mathrm{G}$ network, offers a new range connection e.g., safer transportation system, smart cities, homes, school, smart manufacturing units, etc. [3]. Apart from this, it is believed that the 5G network will offer a better form of connectivity between machine-to-machine and device-to-device. As 5G network offers adoption of machine learning, it is possible to make the network quite smart and decisive with evolving smart appliances. It also offers tangible advantages to society by supporting novel IoT technologies, ubiquitous connection, reduced energy consumption, larger coverage, and smart data management [4]. It offers the capability of massive machine type communication as well as an ultra-reliable communication system with low latency. It is interesting to know that the user plane of $5 \mathrm{G}$ encapsulates the control plane of $4 \mathrm{G}$, which will mean that both $4 \mathrm{G}$ and $5 \mathrm{G}$ work together. It is also known that $5 \mathrm{G}$ offers a completely standalone operation. The frequency of the $5 \mathrm{G}$ network supports less than $1 \mathrm{GHz}$ coverage for device-to-device communication. The complete architecture of the $5 \mathrm{G}$ network is composed of a radio access network as well as the core network. The radio access network comprises of towers, cells that bridges communication between the user with the wireless device and the core network. Basically, the core network will consist of the data network to manage different communication/connection [5]. It is also believed that the core network of $5 \mathrm{G}$ is integrated with cloud-based services as well as the internet in order to offer distributed servers. The complete 5G technology is designed 
on the top of the conventional antenna system (multiple-input-multiple-output), advanced antenna system (beamforming), small cells, millimeter waves, etc.

It is anticipated that the commercial launch of the $5 \mathrm{G}$ network will be carried out by 2020 , and mass adoption will be carried out in 2025. However, the deployment of the 5G network is associated with the following factors like data coverage, ultra-dense network, high data rate with uniform speed, vertical handover, interference issue, etc. [6]. The theoretical claim of the $5 \mathrm{G}$ network system is quite challenging to build, but thousands of dedicated researchers have already started their explorative journey towards investigating the factors leading to the successful deployment of 5G. As 5G is meant for overwhelming connectivity and catering up their demands, it is necessary to ensure that it works in that way. There are various impediments towards this journey. Developing connectivity among the massive number of devices will require innovative planning, comprehensive communication protocol, risk management, re-consideration of various intrinsic and extrinsic constraints, re-consideration of essential parameters associated with the investigation. The prime contribution of this paper is to review some of the significant research work towards improving 5G networks and explore the true scenario or research world of 5G. The paper is organized as: Section II and III discusses essentials of $5 \mathrm{G}$ network followed by a discussion of existing research approaches towards solving problems in the 5G network. Section IV discusses research approaches towards $5 \mathrm{G}$ network using cognitive radio networks. Section V discusses research findings, while Section VI discusses the conclusion and future work.

\section{ESSENTIALS OF 5G NETWORK SYSTEMS}

It is said that there will be an exponential use of mobile networks by 2020 owing to the growth of application supporting Internet-of-Things (IoT) [7]. The prime targets to be achieved in 5G communications will be basically faster data speed, low latency, less power consumption, efficient bandwidth usage, etc. In the present time, the IoT network is sustained using existing wireless system e.g., IEEE 802.11x, 3/4G, etc. which is never enough to cater up the needs of communication supported by IoT devices. Hence, the 5G network is mainly meant for catering up to the communication requirements of large scale IoT system. Fig.1 shows the speed that is offered by different generations and $5 \mathrm{G}$.

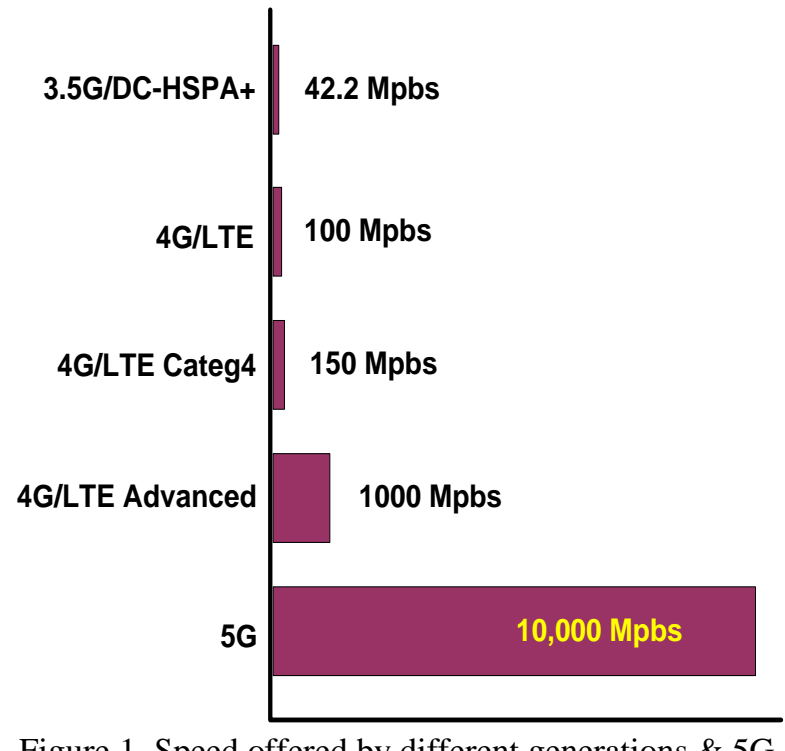

Figure 1. Speed offered by different generations \& 5G

There are 8 technical specifications of $5 \mathrm{G}$ network system i.e., i) it should offer $99.99 \%$ of data/service availability, ii) it should offer $100 \%$ of coverage towards various heterogeneous devices, iii) it should offer $90 \%$ of reduction in usage of network energy, iv) it should ensure 10 years of network lifetime for all the energy-constraint IoT communicating device, v) it should offer connectivity to 100 times of wireless devices as compared to the existing techniques, vi) it should offer 1000 times of channel capacity per unit area to all the IoT devices, vii) it should offer no more than 1 millisecond of latency, and viii) it should offer up to 10 Gbps of data rate which is basically 10-100 times better than existing approaches. It should be noted that the $5 \mathrm{G}$ network is essentially meant for increasing the broadband wireless services for the mobile network over IoT and other critical systems. The operation of the $5 \mathrm{G}$ is investigated with respect to different forms of multiple access technologies as follows:

- Orthogonal Frequency Division Multiple Access (OFDMA): This multiple access scheme was claimed for higher success rate over 4G deployments; however, the networking communities are also claiming for 
their possible deployment over the $5 \mathrm{G}$ network too [8-10]. The prime reason behind this consideration is that it is independent of OFDM deployment.

- $\quad$ Sparse Code Multiple Access (SCMA): This multiple schemes retains the benefits of both OFDMA and CDMA scheme [10][11].By appending a spreading code to the carrier, it offers transmission capability to a higher level. It is also capable of retaining a better form of spectral efficiency level.

- Non-Orthogonal Multiple Access (NOMA): This multiple access scheme is designed using OFDMA and OFDM with a discrete Fourier transform. This technique is claimed to offer better power control scheme in $5 \mathrm{G}$.

However, there is no standard report to claim that which one out of the above three access scheme is the best. The radio access of $5 \mathrm{G}$ comprises of New Radio and Long Term Evolution [12]. At present, there are various existing systems that are formed on the basis of cyclic prefixed-OFDM scheme or CP-OFDM. Some examples are filtered-OFDM [13], Pulse Shaped OFDM [14], Windowed OFDM [15], Unique Word OFDM [16], etc. The existing system has also formulated some complex waveforms to redesign the radio access e.g., Filter-Bank Multi-Carrier waveforms [17]. According to Zaidi et al. [12], CP-OFDM is the most eligible candidate radio access for $5 \mathrm{G}$. However, this is an ongoing discussion and will consume more time to ascertain the fact. The challenges involved in construction $5 \mathrm{G}$ technology are i) good supportability of multiple-inputmultiple-output, ii) smart usage of spectrum, iii) Fixed Wireless Access, iv) instantaneous deployment of dynamic architecture, v) retain the same cost and increase network capacity, vi) security. The root causes of all these problems are associated with resource allocation factor, which is basically a technique to maximize the efficiency of energy in order to assign effect radio resources. This operation results in increasing energy conservation. Apart from resource allocation, network planning is another significant challenge in 5G deployment. The deployments of the nodes are carried out in such a way that coverage is maximized for all the nodes. Energy harvesting is another essential process that is highly challenging to meet. The primary problems associated with the 5G deployment are basically i) the requirement of the holistic methods, ii) dealing with the network interference, iii) dealing with the arbitrariness. The technological challenges associated with the 5G deployment will be i) interference between same cells, ii) efficient Medium Access Control, iii) effective traffic management [18]. Apart from these, there are also many other challenges that are also difficult to be addressed i.e., multiple services, security, and privacy factor, infrastructure, communication, and sensing.

From the application viewpoint, $5 \mathrm{G}$ is targeted to support high-speed mobile networks, multimedia, and entertainment, Internet-of-Things (smart home, logistic, smart shipping, smart cities, industrial application, automation, smart farming, fleet management, healthcare and mission-critical application, autonomous driving, drone operation, security, and surveillance system). However, these applications are yet within the boundaries of the research arena of $5 \mathrm{G}$, and yet it will take more time to see it in real-time. However, there are some critical problems that are yet to be solved in order to see applications of $5 \mathrm{G}$ in reality.

\section{EXISTING TECHNIQUES OF 5G}

Irrespective of the claimed, as well as its advantage, the practical usage of $5 \mathrm{G}$ technologies, is highly challenging. From commercial usage, still its implementation is shrouded by various pitfalls viz. i) $5 \mathrm{G}$ targets mainly on supporting IoT communication system however, it doesn't offer a robust signaling minimization of control plane to users as well as it does offer much good access control practices, ii) 5G technology doesn't claim of its capability to handle massive volume of data over mobile networks, iii) trade-off between the operating cost and need of exponential rise of data capacity, iv) lack of architecture that offers faster service deployment, v) unreliable supports to critical services, vi) non supportability on dynamic augmented reality, vii) less supportability on device-to-device communication system, and viii) usage of old and unsupportive access scheme. It has also been seen that there are three core problems in the 5G network that are all interconnected with each other and are yet unsolved. This section highlights some of the critical problems as well as existing solutions towards addressing these problems:

\subsection{Solutions towards Energy Problems}

Energy is one of the core problems associated with $5 \mathrm{G}$ technologies, and there is various research work that has already reviewed different solutions to resist unwanted energy draining from a different perspective. In reality, the problems associated with energy is solved using different approaches e.g., resource allocation, deployment \& planning, hardware solution, and energy harvesting [19] 


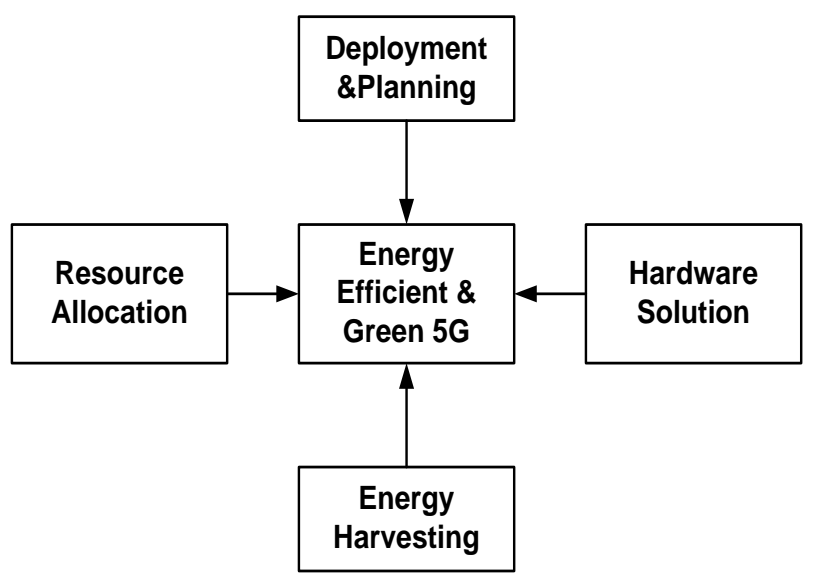

Figure 2. Existing Approaches towards energy problems [19]

Literature has a discussion of architectures with supportability of energy efficiency in 5G networks. The discussion carried out by Abrol et al. [20] has emphasized energy problems where it was discussed that the selection of an external relay node could assist in energy efficiency. Optimization is another process for ensuring a better degree of energy efficiency in 5G networks. The work carried out by Cheng et al. [21] have used a statistical approach in order to emphasize power efficiency considering both single input and multiple input forms of the signal improving the throughput. The work of Reyes et al. [22] has discussed the impact of aggregation of the carrier within efficient bandwidth resulting in energy efficiency. The work carried out by $\mathrm{Zi}$ et al. [23] has offered an optimization principle on energy efficiency problems considering chains of radio frequency. The study has used the millimeter wave and iterative precoding operation in order to save energy.

It was also discussed that the devices used in $5 \mathrm{G}$ networks are too much depending on charging operations in order to boost up maximum residual energy. However, it also demands a high grade of synchronization with other nodes in the mobile network too. A new conceptualized problem of charging the mobile devices over 5G has been evolving in the existing system. The recent research work carried out by Ai et al. [24] has introduced a distributed algorithm for assisting in mobile charging using an energy transfer process that also promotes the capacity of the network. The assessment of the study shows lesser dependencies of chargers with an increase of communicating devices. The similar direction of charging technology was also put forward by Galinina et al. [25]. Apart from the charging-based concept, energy harvesting is also found to be used for boosting the energy performance of $5 \mathrm{G}$ networks. Usage of Beamforming is another practice to control energy consumption in $5 \mathrm{G}$ networks. Approaches like power transfer over macro-cell were proven to be improved using harvestingbased approaches. The study carried out by Sheng et al. [26] have used fractional programming for developing zero forcings and mixed Beamforming. According to the recent study of Huang et al. [27], energy harvesting over software-defined radio architectures offers better energy efficiency. The authors have applied a stochastic approach for computing the level of energy. The allocation mechanism of power also assists in solving energy problems. However, dynamic allocation offers much better benefits to deal with uncertain traffic. This problem was addressed by AlQerm [28] where a learning mechanism has been discussed in order to enhance the dynamic power allocation, especially for multi-tier networks. Energy problems have also been investigated with respect to the reconfigurable networks owing to maximum power consumption on them. Study towards such issues concerning of usage of the radio access network and the optical network was carried out by Fiorani et al. [29]. The study findings suggest that transport network leads to lots of power consumption that can be resisted using radio networks and transport network together. Study towards the usage of transport network was also emphasized by Wong et al. [30] where a ring network was chosen for energy efficiency. Another recent trend of wideband broadcasting is found to offer control over the power consumption over devices working in $5 \mathrm{G}$ networks. This concept controls power consumption by defining frequency reuse. According to Gimenez et al. [31], this concept assists in practical frequency reuse for potential power saving.

\subsection{Solution towards Resource Allocation Problem}

The $5 \mathrm{G}$ network is actually meant for supporting IoT application where massive data, as well as service offerings, will be made. In order to offer a QoS based communication system, it is necessary to ensure that resource allocation problem is mitigated or addressed in an effective manner. By allocation the precise amount of network/computational resource, it is feasible for maintaining better communication performance with lower wastage of resources. Irrespective of various research work towards resource allocation over the ubiquitous network [32]-[35], the problem is very different in the case of the 5G networks where millions heterogeneous devices will be connected using different communication protocols [36]. 
Resource allocation problem is closely linked with the transmission patterns depending upon the usage of the relay channel. This problem was studied by Chen et al. [37] who have introduced a decode-forwarding scheme for improving the relay performance. The study outcome exhibits better bandwidth usage with efficient power utilization. Downlink scheduling is another essential factor controlling the resource allocation performance within the device running on $5 \mathrm{G}$ networks. However, there is quite a contradiction of usage of OFDM and FBMC to be restricted over physical layers only. Therefore, this issue was investigated by Femenias et al. [38] who have presented a cross-layered model for improving the scheduling performance of 5G networks. The study has also used different forms of performance parameters over challenging networks using greedy-based approach. The study has also proved that hybridization of OFDM could offer better result compared to the legacy version of OFDM. It was also seen that the selection of mode for assisting in better transmission form also helps in a better form of resource allocation in device-to-device networks. A study carried out by Jiang et al. [39] have introduced a scheme to allocate resource considering the mode selection process. The study outcome shows that switching operation is highly affected by the presented technique. More recently, a discussion presented by Feng et al. [40] has stated that $5 \mathrm{G}$ will encourage the exponential usage of social network with a more massive number of devices. Therefore, managing the dynamic contents is a highly problematic task in this perspective. Therefore, the study has presented a content sharing mechanism that is capable of performing resource allocation. Social aware factor has also been considered in the study of Zhao et al. [41] that is meant for performing multicast communication for effective power control.

In a similar line of research, it was found that quality of service is very hard to maintain in resource allocation process in IoT communication system using 5G. It was discussed by Gandotra et al. [42] that usage of a sectored antenna will significantly boost the QoS performance in IoT communication system. However, such a scheme doesn't assist in using common resource existing over different cells. Therefore, the work carried out by Huang et al. [43] has presented a game-based model to improve the performance of resource allocation and address this problem. The game-based approach was also discussed by $\mathrm{Yu}$ et al. [44] who have used nonlinear complementary approach for integrating mobile networks with a software-defined network.

Most recently, resource allocation was also investigated with respect to the energy factor over IoT. Li et al. [45] have developed a mechanism where the resource allocation is studied with respect to power and channel. The authors have presented a solution using game theory, and its outcome was claimed to cater up the QoS demands. A unique mechanism for resource allocation has been developed by Liu et al. [46] where nonorthogonal multiple access has been adopted for accelerating the transmission rate in $5 \mathrm{G}$ networks. The system is capable of resisting all sorts of channel variation occurring due to constraint over hardware design. A collaborative approach was designed for improving relaying performance. Usage of non-orthogonal multiple access was also investigated by $\mathrm{Wu}$ et al. [47] focusing on boosting up resource allocation performance of IoT system. Apart from the collaborative approach, convex programming is also found to be assistive of resource allocation. The works of Tang et al. [48] have presented a sequential convex programming approach for achieving better admission control over $5 \mathrm{G}$ networks.

\subsection{Solution towards Spectrum Efficiency Problem}

Spectrum efficiency is one of the essential criteria for the standard performance of $5 \mathrm{G}$ networks. Many existing mechanisms make use of refarming of the existing spectrum for exploring more number of spectrum resources. It is said that spectrum efficiency will take another decade to fulfill the practical demands of existing users. However, the 5G network is claimed to address this problem. The technical claims of a 5G network to offer 1000 times of enhanced throughput with 10 times of spectral efficiency [49]. There are various problems associated with improving spectral efficiency. Spectrum sharing is one of the core processes in improving spectrum efficiency. The study carried out by Ercan et al. [50] has discussed the impact of network utilization in IoT over the gain of multi-user. The study outcome discussed all the dependency factors for improving the spectral efficiency in 5G. Network density is another factor that affects the spectrum efficiency to a large extent. The work of Koudouridis et al. [51] has presented an integrated framework that uses both frequency spectrum as well as network density for optimizing the performance of network spectrum efficiency. Apart from this, channel hopping is another process to boost up the performance. The work discussed by Li et al. [52] has a discussion of the channel hopping protocols for assisting in dynamic spectrum management. Study towards a similar approach is carried out by Tsiropoulos et al. [53] where the cooperative framework is discussed for better support of heterogeneous network on 5G. Some more approaches on spectrum efficiency along with energy efficiency consideration were presented by Wu et al.[54], Wu et al. [55], and Zhou [56]. 
Table 1. Summary of Existing Research on 5G

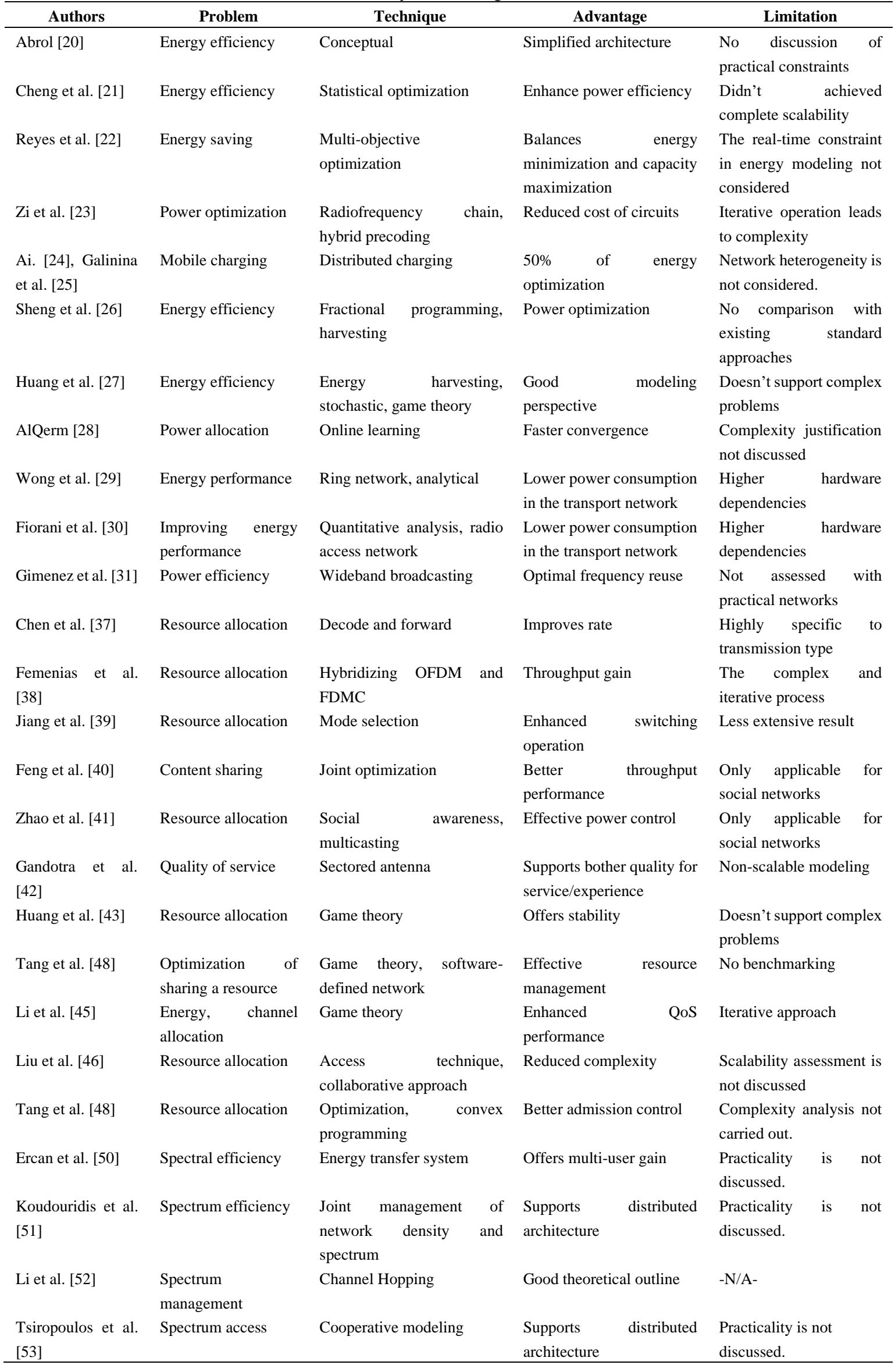




\begin{tabular}{lllll}
\hline Wu et al. [54] & Spectrum efficiency & Analytical approach & $\begin{array}{l}\text { Balances both energy and } \\
\text { spectrum }\end{array}$ & $\begin{array}{l}\text { Practicality is not } \\
\text { discussed. }\end{array}$ \\
Wu et al. [55] & Spectral efficiency & Simulation-based & Good theoretical outline & $\begin{array}{l}\text { Practicality is not } \\
\text { discussed. }\end{array}$ \\
Zhou et al. [56] & Spectral efficiency & $\begin{array}{l}\text { Non-orthogonal access } \\
\text { scheme, radio networks }\end{array}$ & Improves efficiency & No benchmarking \\
\end{tabular}

\section{RESEARCH TRENDS IN 5G}

From the prior section, it can be seen that there is enough effort that has been put forward by the various researcher in order to address the potential problems associated with 5G networking system. The existing solution is yet not optimal, and more effort is required for further making the 5G suitable for offering QoSbased performance to the IoT system. The major problem associated with the 5G networking system is spectrum utilization owing to the growing rate of mobile users as well as services. The dynamic traffic results in spectrum holes where the spectrum is subjected to either over or under-utilization. This problem was found to be significantly addressed by cognitive radio network by autonomously identifying such spectrum holes that offer a better form of spectrum management [57]. Another interesting part of the cognitive radio networks is that it can offer more capabilities to the networking system as it utilizes varied principles of optimization techniques, game theory, genetic algorithm, machine learning, etc. This adoption of diverse techniques significantly assists in offering effective resource optimization. Another essential feature of the cognitive radio network is that it offers its cognitive cycle to build up by learning the network condition in real-time effectively. Moreover, it uses two forms of open system interconnection layer, where the first one is allocated for cognitive medium access control and another for MAC. For the purpose of offering optimal networking performance, cognitive radio network carries out a higher degree of interaction among the layers. The architectural view of cognitive radio is shown in Fig.3. Using this architecture, the cognitive radio assists in leasing all sorts of under-utilized bands of frequency by ensuring zero harmful effects of interference. It is also usual that such forms of the leased spectrum are usually not trusted and is highly opportunistic. This also results in an anticipated reduction in leasing cost in contrast to the purchasing cost of any licensed band. Therefore, this architecture is utilized to offer cognitive radio to lease additional spectrum apart from that offered in licensed bands. The architecture of cognitive radio used over the 5G network is basically classified into two forms viz. i) non-cooperative architecture and ii) cooperative architecture [57]. Both the types of 5G cognitive radio architecture emphasizes on the bandwidth as well as power utilization. However, there are many possible scenarios where the level of resource availability significantly controls the power as well as the bandwidth factor in communication performance of $5 \mathrm{G}$ networks. Another essential parameter that cognitive radio introduces in the 5G network system is its usage of the antenna. Normally, the antenna used in the 5G network system has various problems that are connected with a small aperture as well as array design and impedance bandwidth. There have been various research works where researchers have presented various solutions to improve the antenna design principle.

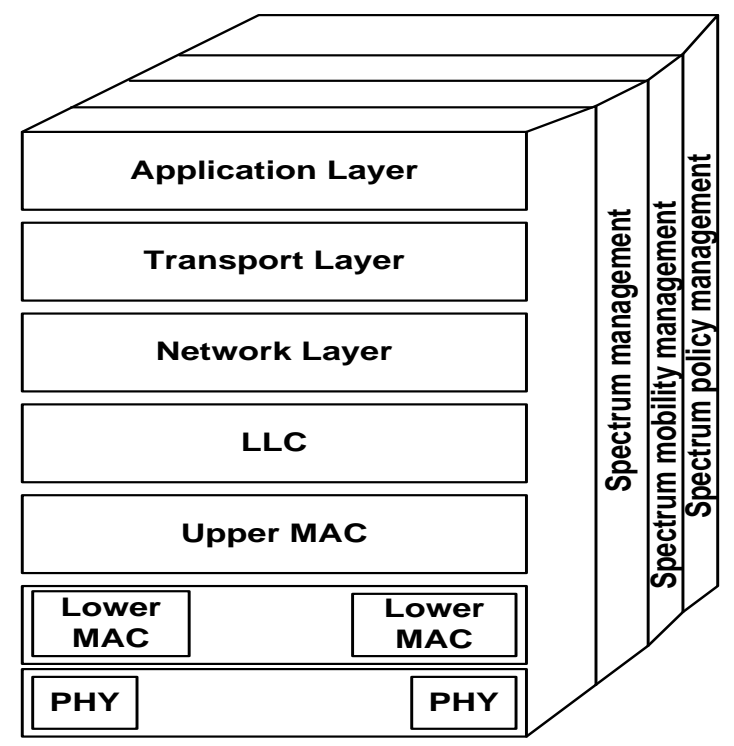

Figure 3. Cognitive Radio Architecture 
Hence, it is necessary to understand the effectiveness of the approaches presented by various researchers adopting a cognitive radio network. This section also reviews all the existing approaches and its successful and limiting factors with respect to potential problems over $5 \mathrm{G}$ network systems.

\subsection{Solution towards Energy Problems}

Owing to the efficient balancing capability of the cognitive radio network, it is expected to solve the problem associated with energy consumption in the 5G network. Power allocation in an optimal manner significantly assists in improving the performance of the cooperative network in 5G. The work discussed by Diamantoulakis et al. [58] has emphasized this fact and has presented a technique to select the relay node in a cooperative network of cognitive radio system. The study implements amplify and forward as well as decode and forward mode for performing relay operation. Convex optimization approach was implemented for this purpose. With a similar direction to the problem, it was also investigated that spectrum sharing is highly influenced by the energy factors involved in cognitive radio nodes in the $5 \mathrm{G}$ system. The research work of Mach and Becvar [59] has presented a centralized method that is capable of sharing the spectrum with a target of minimizing the energy consumption at the small cell base station. The study also reduces the overhead as well as control the complexity to a large extent. Just like conventional power transfer scheme, there is a certainly improved technique that uses energy harvesting mechanism. Such a study is carried out by Verma et al. [60] where decode and forward scheme has been used for energy saving in cognitive radio network over the 5G system. A feedback-scheme is developed in the study that takes input of all the energy readings to show that the presented system offers higher network capacity. The density of the network in 5G also affects the energy consumption of the devices considerably. The study carried out by Yang et al. [61] has used a game theory for increasing the energy efficiency associated with dense networks in 5G. Access technology also influences the energy efficiency. This fact was investigated by very less number of researchers. The study carried out by Zhai et al. [62] has proved that usage of orthogonal frequency division multiple accesses as well as sparse code multiple accesses. The study has presented an integrated resource management scheme that is capable of reducing the transmit power for the device-to-device communication system.

\subsection{Solution towards Resource Allocation Problem}

The approaches used for addressing resource allocation problem in $5 \mathrm{G}$ were quite high as compared to that using cognitive radio network. With the adoption of the cognitive radio network, it has been seen that it evolves up with a new problem of optimization that is related to computation of least number of the channel to be identified and send by the devices in $5 \mathrm{G}$ networks. This problem was found to be addressed by Ejaz and Ibnkahla [63] by introducing a cross layer-based modeling for assisting in dynamic allocation of resources for IoT networks. The study outcome was found to be further improved by applying game theory over the crosslayer scheme in order to fulfill the QoS requirement. Another work carried by Liu et al. [64] has investigated the impact of using non-orthogonal multiple access schemes over the $5 \mathrm{G}$ network for assessing resource optimization performance. The study outcome has shown a reduction in energy consumption and enhanced transmission performance.

\subsection{Solution towards Spectral Efficiency Problem}

It is already known that usage of the cognitive radio network is basically meant for addressing the spectral efficiency problem that normal approach is incapable of. However, the rate of research work in this direction is quite less. Usage of sparse access policy was claimed to offer better accessibility over $5 \mathrm{G}$ networking system. This claim was investigated by Hosseini et al. [65] where an adaptive policy was introduced using wavelets for precise frequency estimation. The study outcome was found to offer better accuracy with less error. The evidence-based theory was another implementation-based technique in order to enhance spectral efficiency. The work carried out by Liu et al. [66] has used the Dempster-Shafer principle for assisting in multi-modal sensing method. The study outcome was claimed to offer to enhance sensing performance in the presence of challenging channel condition. The work carried out by Song et al. [67] has used the optimization-based principle in order to balance the better performance of spectrum and resource allocation. The mechanism of joint resource allocation is found to improve spectral sharing by enhancing non-orthogonal multiple access policies significantly. 
Table.2 Summary of Existing Research on 5G using Cognitive Radio Networks

\begin{tabular}{|c|c|c|c|c|}
\hline Authors & Problem & Technique & Advantage & Limitation \\
\hline $\begin{array}{l}\text { Diamantoulakis et } \\
\text { al. [58] }\end{array}$ & Power allocation & Convex optimization & Increased rate & Highly iterative process \\
\hline $\begin{array}{l}\text { Mach and Becvar } \\
\text { [59] }\end{array}$ & Energy awareness & $\begin{array}{l}\text { The centralized } \\
\text { scheme, distributed, a } \\
\text { section of small cell } \\
\text { base station }\end{array}$ & Low overhead & Narrowed study scope \\
\hline Verma et al. [60] & Energy efficiency & $\begin{array}{l}\text { Feedback-scheme } \\
\text { enhanced decode and } \\
\text { forward }\end{array}$ & $\begin{array}{l}\text { Increased } \\
\text { capacity }\end{array}$ & $\begin{array}{l}\text { Less emphasis } \\
\text { transmittance power }\end{array}$ \\
\hline Yang et al. [61] & $\begin{array}{l}\text { Maximizing energy } \\
\text { efficiency }\end{array}$ & Game theory & Improved fairness & Lesser extensive analysis \\
\hline Zhai et al. [62] & $\begin{array}{l}\text { Impact of access } \\
\text { technology on energy }\end{array}$ & $\begin{array}{l}\text { Integrated resource } \\
\text { management }\end{array}$ & $\begin{array}{l}\text { Enhanced network } \\
\text { capacity }\end{array}$ & Lesser extensive analysis \\
\hline $\begin{array}{l}\text { Ejaz and Ibnkahla } \\
{[63]}\end{array}$ & Resource allocation & Game theory & $\begin{array}{l}\text { Better control of energy } \\
\text { reduction }\end{array}$ & $\begin{array}{l}\text { Cannot solve complex } \\
\text { network problems }\end{array}$ \\
\hline Liu et al. [66] & Spectrum sensing & Dempster-Shafer & $\begin{array}{l}\text { Improved } \\
\text { performance }\end{array}$ & $\begin{array}{l}\text { Narrowed study scope, less } \\
\text { practical approach. }\end{array}$ \\
\hline Song et al. [67] & Spectrum utilization & $\begin{array}{l}\text { Optimization } \\
\text { approach }\end{array}$ & $\begin{array}{l}\text { Effective } \\
\text { sharing }\end{array}$ & $\begin{array}{l}\text { Involves computational } \\
\text { complexity and is not energy } \\
\text { efficient }\end{array}$ \\
\hline
\end{tabular}

\section{CORE RESEARCH FINDINGS}

After reviewing the existing approaches (normal approaches and cognitive radio network based approaches) for improving the communication performance of $5 \mathrm{G}$ networking system, it is found that there are potential breakthrough findings as well as potential research problems. Both the information is equally important in order to formulate a new study towards addressing similar problems.

\subsection{Core Potential}

It has been seen that there are good practice of computational model like game theory (Ejaz and Ibnkahla [63], Li et al. [45] Huang et al. [27], Tang et al. [48], Yang et al. [61]), analytical modeling (Cheng et al. [21], Sheng et al. [26], Gimenez et al. [31], Tsiropoulos et al. [53], Wu et al. [54]), evidence theory (Liu et al. [66]) etc. There are various models that have been proven to offer better decision making concepts that are useful for exploring the true cause of problem associated with the power problem, resource allocation problem, and spectral efficiency problem in proposed techniques. Various optimization principles (Reyes et al. [22], Feng et al. [40], etc.) are also found to be testified for both the existing approaches on a 5G network system, which opens up the avenue of research towards using cost-factor controlling on $5 \mathrm{G}$ device utilization. There are also existing approaches that supports different forms of forwarding scheme (Verma et al. [60], Chen et al. [37], etc) as well as access scheme (Liu et al. [64], Zhou et al. [56], etc) which also gives an interesting idea that future work must be carried out towards these scheme for an alternative means of energy saving as well as spectral efficiency. However, all these schemes are associated with the specific case study as well as beneficial points that is not totally applicable to real-time devices with practical constraints over the $5 \mathrm{G}$ network.

\subsection{Research Gap}

The research gap will mean that there exist certain problems in existing systems (out of many problems) which are highly essential to be addressed in order to pave the path of effective research direction towards 5G. The important points of the research gap are as follows:

- A lesser study using Cognitive Radio Network: The data collected from known publications like IEEE, Springer, Elsevier, Hindawi, etc., by providing the keyword of Cognitive radio + resource allocation + energy + spectral efficiency and conventional approach. Based on these data, it is observed that researched published on conventional approaches are higher than that of cognitive approaches. It has been theoretically expressed everywhere the beneficial part of using cognitive radio networks on the 5G system. However, in reality, the facts are quite different. A closer look into the existing trend (Fig.4) shows that there is very less number of research work is focused on towards adoption cognitive radio network in spite of its reported advantages in $5 \mathrm{G}$. 


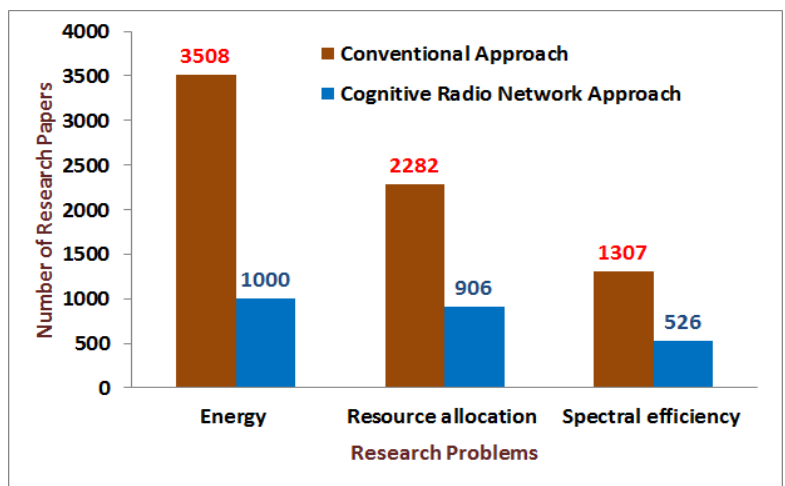

Figure 4. Research Trend of existing Approaches in 5G

- The tradeoff between Optimization and Actual Demands: Optimization is perhaps one of the best approaches towards developing a model addressing the problems associated with $5 \mathrm{G}$ networks. However, the existing optimization-based approach is highly recursive as well as iterative with a target to obtain the best result but at the cost of computational complexity. Hence, such optimization approaches are less progressive and more iterative in nature where the iteration is controlled by the hard-thresholding mechanism. Such approaches can be theoretically sound but are not practical.

- Ignorance of Constraints in Modeling: It was also seen that there are various approaches that are repeatedly used e.g., game theory. Usage of such an approach can only offer a symptomatic solution and doesn't address the full problem as they never consider probability as well as they never consider any form of real-time constraint or risk associated with 5G networks. Existing approaches using non-orthogonal access policies delivered better performance using, but they have higher dependencies over pre-defined information of channel gain from the base station, which is not practical. A similar problem is also associated with the sparse code multiple access schemes i.e.; they are dependent over predefined information of sparsity. Such kinds of information are not feasible to be given in dynamic networks like IoT in $5 \mathrm{G}$ networks.

- Lack of consideration of real parameters: It is well known that $5 \mathrm{G}$ was designed to support IoT that works on the basis of sensor and multiple-input-multiple-output. At present, there are no more studies that define the traffic level optimization considering these parameters that offer a true simulating environment to perform analysis of $5 \mathrm{G}$ with respect to resource allocation, energy problems, spectral efficiency, etc.

\section{CONCLUSION}

From the discussion presented in this review work, it is attempted to highlight that in order to retain the theoretical claim of a $5 \mathrm{G}$ network, the cognitive radio network is highly essential as well as mandatory. Adoption of a cognitive radio network is the only mechanism with the help of which it is feasible to offer a better form of spectral efficiency. From the existing patterns of approaches, it is understood that researchers have classified their approaches towards enhancing the $5 \mathrm{G}$ communication performance into three types viz., approaches towards solving energy problems, resource allocation problems, and spectral efficiency problem. However, it is also clear from the discussion that all these problems are highly linked with each other and isolated mechanism of addressing such problems will never offer a full fledged solution. The paper has also highlighted the potential as well as limiting features of the existing approaches that assist the readers in concluding the exact scenario and will assist them in shaping their implementation ideology likewise.

The future work will be in the direction of bridging the research gap. The first initiative could be taken towards considering a highly distributive and heterogeneous $5 \mathrm{G}$ network with cognitive radio focusing on resource efficiency as well as optimal allocation. The second initiative of future work will be carried out towards addressing the problem associated with understanding and realizing the dynamic size of incoming traffic. This will assist in solving the scheduling as well as resource allocation problem, which will also indirectly assists in energy saving and spectral efficiency. The throughput could be enhanced by working with the multiple-input-multiple-output principle in a cognitive radio network with a sole motive of cost-effective optimization.

\section{REFERENCES}

Insights on Significant Implication on Research Approach for Enhancing 5 G Network System (Vani BP, et al) 
[1] “Road to 5G,” Academic Press, 19-Jul-2016

[2] Verma, Jyoti, Sunanda Gupta, Pratima Manhas, and Vasudha Arora. "Target Network Selection Algorithm based on Required Dwell Time Estimation." Indonesian Journal of Electrical Engineering and Informatics (IJEEI) 6, no. 2 (2018): 172-182.

[3] S. Asif, "5G Mobile Communications: Concepts and Technologies," CRC Press, 26-Jul-2018 - Computers

[4] S. Sen, L. Datta, S. Mitra, "Machine Learning and IoT: A Biological Perspective," CRC Press, 04-Jul-2018 Computers

[5] Kumari, Sunita, and S. Y. Kumar. "Design Analysis and Development of Inverter Topologies for Industries." Indonesian Journal of Electrical Engineering and Informatics (IJEEI) 6, no. 1 (2018): 53-60.

[6] Ramjee Prasad, "5G: 2020 and Beyond,” River Publishers, 01-Sep-2014

[7] S. Jaloudi, "A bridge between legacy wireless communication systems and internet of things." Indonesian Journal of Electrical Engineering and Informatics (IJEEI) 7, no. 2 (2019): 279-288.

[8] Tao Jiang, Lingyang Song, Yan Zhang, “Orthogonal Frequency Division Multiple Access Fundamentals and Applications," CRC Press, 21-Apr-2010

[9] HrishikeshVenkataraman, Ramona Trestian, "5G Radio Access Networks: Centralized RAN, Cloud-RAN and Virtualization of Small Cells," CRC Press, 16-Mar-2017

[10] Wei Xiang, KanZheng, Xuemin (Sherman) Shen, 5 G Mobile Communications, Springer, 13-Oct-2016

[11] AfifOsseiran, Jose F. Monserrat, Patrick Marsch, "5G Mobile and Wireless Communications Technology," Cambridge University Press, 02-Jun-2016

[12] A. A. Zaidi et al., "Waveform and Numerology to Support 5G Services and Requirements," in IEEE Communications Magazine, vol. 54, no. 11, pp. 90-98, November 2016

[13] Y. Qiu, Z. Liu and D. Qu, "Filtered bank based implementation for filtered OFDM," 2017 7th IEEE International Conference on Electronics Information and Emergency Communication (ICEIEC), Macau, 2017, pp. 15-18.

[14] O. Narmanlioglu, R. C. Kizilirmak and M. Uysal, "The impact of pulse shaping filters on OFDM-based visible light communications," 2016 IEEE 10th International Conference on Application of Information and Communication Technologies (AICT), Baku, 2016, pp. 1-4

[15] L. Yang and Y. Xu, "Filtered-OFDM system performance research based on Nuttall's Blackman-Harris window," 2017 IEEE 17th International Conference on Communication Technology (ICCT), Chengdu, 2017, pp. 687-691.

[16] M. Huemer, C. Hofbauer and J. B. Huber, "Non-Systematic Complex Number RS Coded OFDM by Unique Word Prefix," in IEEE Transactions on Signal Processing, vol. 60, no. 1, pp. 285-299, Jan. 2012.

[17] W. Xu, C. Wang, G. Cui, W. Wang and Y. Zhang, "The M-sequence encoding method for a radar-communication system based on filter bank multi-carrier," 2017 9th International Conference on Advanced Infocomm Technology (ICAIT), Chengdu, 2017, pp. 255-259.

[18] António Morgado, Kazi Mohammed Saidul Huq, Shahid Mumtaz, Jonathan Rodriguez,"A survey of 5G technologies: regulatory, standardization and industrial perspectives", ScienceDirect, Digital Communications and Networks, Volume 4, Issue 2, April 2018, Pages 87-97

[19] S. Buzzi, C. I, T. E. Klein, H. V. Poor, C. Yang and A. Zappone, "A Survey of Energy-Efficient Techniques for 5G Networks and Challenges Ahead," in IEEE Journal on Selected Areas in Communications, vol. 34, no. 4, pp. $697-$ 709, April 2016

[20] A. Abrol and R. K. Jha, "Power Optimization in 5G Networks: A Step Towards GrEEn Communication," in IEEE Access, vol. 4, pp. 1355-1374, 2016

[21] W. Cheng, X. Zhang and H. Zhang, "Statistical-QoS Driven Energy-Efficiency Optimization Over Green 5G Mobile Wireless Networks," in IEEE Journal on Selected Areas in Communications, vol. 34, no. 12, pp. 3092-3107, Dec. 2016

[22] E. Chavarria-Reyes, I. F. Akyildiz and E. Fadel, "Energy-Efficient Multi-Stream Carrier Aggregation for Heterogeneous Networks in 5G Wireless Systems," in IEEE Transactions on Wireless Communications, vol. 15, no. 11, pp. 7432-7443, Nov. 2016.

[23] R. Zi, X. Ge, J. Thompson, C. Wang, H. Wang and T. Han, "Energy Efficiency Optimization of 5G Radio Frequency Chain Systems," in IEEE Journal on Selected Areas in Communications, vol. 34, no. 4, pp. 758-771, April 2016.

[24] Z. Ai, Y. Liu, F. Song and H. Zhang, "A Smart Collaborative Charging Algorithm for Mobile Power Distribution in 5G Networks," in IEEE Access, vol. 6, pp. 28668-28679, 2018.

[25] O. Galinina, H. Tabassum, K. Mikhaylov, S. Andreev, E. Hossain and Y. Koucheryavy, "On the feasibility of 5Ggrade dedicated RF charging technology for wireless-powered wearables," in IEEE Wireless Communications, vol. 23, no. 2, pp. 28-37, April 2016.

[26] M. Sheng, L. Wang, X. Wang, Y. Zhang, C. Xu and J. Li, "Energy Efficient Beamforming in MISO Heterogeneous Cellular Networks With Wireless Information and Power Transfer," in IEEE Journal on Selected Areas in Communications, vol. 34, no. 4, pp. 954-968, April 2016.

[27] X. Huang et al., "Software Defined Energy Harvesting Networking for 5G Green Communications," in IEEE Wireless Communications, vol. 24, no. 4, pp. 38-45, Aug. 2017.

[28] I. AlQerm and B. Shihada, "Energy-Efficient Power Allocation in Multitier 5G Networks Using Enhanced Online Learning," in IEEE Transactions on Vehicular Technology, vol. 66, no. 12, pp. 11086-11097, Dec. 2017

[29] M. Fiorani, S. Tombaz, J. Martensson, B. Skubic, L. Wosinska and P. Monti, "Modeling energy performance of CRAN with optical transport in 5G network scenarios," in IEEE/OSA Journal of Optical Communications and Networking, vol. 8, no. 11, pp. B21-B34, Nov. 2016.

[30] E. Wong, E. Grigoreva, L. Wosinska and C. M. Machuca, "Enhancing the survivability and power savings of 5G transport networks based on DWDM rings," in IEEE/OSA Journal of Optical Communications and Networking, vol. 9, no. 9, pp. D74-D85, Sept. 2017. 
[31] J. J. Gimenez, D. Gomez-Barquero, J. Morgade and E. Stare, "Wideband Broadcasting: A Power-Efficient Approach to 5G Broadcasting," in IEEE Communications Magazine, vol. 56, no. 3, pp. 119-125, MARCH 2018.

[32] Y. Teng, M. Liu, F. R. Yu, V. C. M. Leung, M. Song and Y. Zhang, "Resource Allocation for Ultra-Dense Networks: A Survey, Some Research Issues and Challenges," in IEEE Communications Surveys \& Tutorials.

[33] R. O. Afolabi, A. Dadlani and K. Kim, "Multicast Scheduling and Resource Allocation Algorithms for OFDMABased Systems: A Survey," in IEEE Communications Surveys \& Tutorials, vol. 15, no. 1, pp. 240-254, First Quarter 2013.

[34] O. Yazdani and G. Mirjalily, "A survey of distributed resource allocation for device-to-device communication in cellular networks," 2017 Artificial Intelligence and Signal Processing Conference (AISP), Shiraz, 2017, pp. 236-239.

[35] T. A. Xavier and R. Rejimoan, "Survey on various resource allocation strategies in cloud," 2016 International Conference on Circuit, Power and Computing Technologies (ICCPCT), Nagercoil, 2016, pp. 1-4.

[36] G. A. Akpakwu, B. J. Silva, G. P. Hancke and A. M. Abu-Mahfouz, "A Survey on 5G Networks for the Internet of Things: Communication Technologies and Challenges," in IEEE Access, vol. 6, pp. 3619-3647, 2018.

[37] Z. Chen, T. Li, P. Fan, T. Q. S. Quek and K. B. Letaief, "Cooperation in 5G Heterogeneous Networking: Relay Scheme Combination and Resource Allocation," in IEEE Transactions on Communications, vol. 64, no. 8, pp. 3430-3443, Aug. 2016.

[38] G. Femenias, F. Riera-Palou, X. Mestre and J. J. Olmos, "Downlink Scheduling and Resource Allocation for 5G MIMO-Multicarrier: OFDM vs FBMC/OQAM," in IEEE Access, vol. 5, pp. 13770-13786, 2017.

[39] F. Jiang, B. Wang, C. Sun, Y. Liu and R. Wang, "Mode selection and resource allocation for device-to-device communications in 5G cellular networks," in China Communications, vol. 13, no. 6, pp. 32-47, June 2016.

[40] L. Feng et al., "Resource Allocation for 5G D2D Multicast Content Sharing in Social-Aware Cellular Networks," in IEEE Communications Magazine, vol. 56, no. 3, pp. 112-118, MARCH 2018.

[41] P. Zhao, L. Feng, P. Yu, W. Li and X. Qiu, "A Social-Aware Resource Allocation for 5G Device-to-Device Multicast Communication," in IEEE Access, vol. 5, pp. 15717-15730, 2017.

[42] P. Gandotra, R. K. Jha, S. Jain, "Sector-Based radio Resource Allocation (SBRRA) Algorithm for Better Quality of Serice and Experience in Device-to-Device (D2D) Communication", Cornell University Library, 2018

[43] J. Huang, C. Xing, Y. Qian and Z. J. Haas, "Resource Allocation for Multicell Device-to-Device Communications Underlaying 5G Networks: A Game-Theoretic Mechanism With Incomplete Information," in IEEE Transactions on Vehicular Technology, vol. 67, no. 3, pp. 2557-2570, March 2018.

[44] R. Yu, J. Ding, X. Huang, M. Zhou, S. Gjessing and Y. Zhang, "Optimal Resource Sharing in 5G-Enabled Vehicular Networks: A Matrix Game Approach," in IEEE Transactions on Vehicular Technology, vol. 65, no. 10, pp. 78447856, Oct. 2016.

[45] S. Li, Q. Ni, Y. Sun, G. Min and S. Al-Rubaye, "Energy-Efficient Resource Allocation for Industrial Cyber-Physical IoT Systems in 5G Era," in IEEE Transactions on Industrial Informatics, vol. 14, no. 6, pp. 2618-2628, June 2018.

[46] X. Liu, Y. Liu, X. Wang and H. Lin, "Highly Efficient 3-D Resource Allocation Techniques in 5G for NOMA-Enabled Massive MIMO and Relaying Systems," in IEEE Journal on Selected Areas in Communications, vol. 35, no. 12, pp. 2785-2797, Dec. 2017.

[47] Yali Wu, G. Kang and N. Zhang, "Random access and resource allocation for the coexistence of NOMA-based and OMA-based M2M communications," in China Communications, vol. 14, no. 6, pp. 43-53, 2017.

[48] R. Tang, J. Zhao, H. Qu and Z. Zhang, "User-Centric Joint Admission Control and Resource Allocation for 5G D2D Extreme Mobile Broadband: A Sequential Convex Programming Approach," in IEEE Communications Letters, vol. 21, no. 7, pp. 1641-1644, July 2017.

[49] L. Zhang, M. Xiao, G. Wu, M. Alam, Y. Liang and S. Li, "A Survey of Advanced Techniques for Spectrum Sharing in 5G Networks," in IEEE Wireless Communications, vol. 24, no. 5, pp. 44-51, October 2017.

[50] A. Ö. Ercan, M. O. Sunay and I. F. Akyildiz, "RF Energy Harvesting and Transfer for Spectrum Sharing Cellular IoT Communications in 5G Systems," in IEEE Transactions on Mobile Computing, vol. 17, no. 7, pp. 1680-1694, 1 July 2018.

[51] G. P. Koudouridis and P. Soldati, "Spectrum and Network Density Management in 5G Ultra-Dense Networks," in IEEE Wireless Communications, vol. 24, no. 5, pp. 30-37, October 2017.

[52] A. Li, G. Han, J. J. P. C. Rodrigues and S. Chan, "Channel Hopping Protocols for Dynamic Spectrum Management in 5G Technology," in IEEE Wireless Communications, vol. 24, no. 5, pp. 102-109, October 2017.

[53] G. I. Tsiropoulos, A. Yadav, M. Zeng and O. A. Dobre, "Cooperation in 5G HetNets: Advanced Spectrum Access and D2D Assisted Communications," in IEEE Wireless Communications, vol. 24, no. 5, pp. 110-117, October 2017.

[54] Y. Wu et al., "Green transmission technologies for balancing the energy efficiency and spectrum efficiency trade-off," in IEEE Communications Magazine, vol. 52, no. 11, pp. 112-120, Nov. 2014.

[55] Q. Wu, W. Chen, D. W. K. Ng and R. Schober, "Spectral and Energy-Efficient Wireless Powered IoT Networks: NOMA or TDMA?," in IEEE Transactions on Vehicular Technology, vol. 67, no. 7, pp. 6663-6667, July 2018.

[56] F. Zhou, Y. Wu, R. Q. Hu, Y. Wang and K. K. Wong, "Energy-Efficient NOMA Enabled Heterogeneous Cloud Radio Access Networks," in IEEE Network, vol. 32, no. 2, pp. 152-160, March-April 2018.

[57] X. Hong, J. Wang, C. Wang and J. Shi, "Cognitive radio in 5G: a perspective on energy-spectral efficiency trade-off," in IEEE Communications Magazine, vol. 52, no. 7, pp. 46-53, July 2014.

[58] P. D. Diamantoulakis, K. N. Pappi, S. Muhaidat, G. K. Karagiannidis and T. Khattab, "Carrier Aggregation for Cooperative Cognitive Radio Networks," in IEEE Transactions on Vehicular Technology, vol. 66, no. 7, pp. 59045918, July 2017.

[59] P. Mach and Z. Becvar, "Energy-Aware Dynamic Selection of Overlay and Underlay Spectrum Sharing for Cognitive Small Cells," in IEEE Transactions on Vehicular Technology, vol. 66, no. 5, pp. 4120-4132, May 2017. 
[60] D. K. Verma, R. Y. Chang and F. Chien, "Energy-Assisted Decode-and-Forward for Energy Harvesting Cooperative Cognitive Networks," in IEEE Transactions on Cognitive Communications and Networking, vol. 3, no. 3, pp. 328342, Sept. 2017.

[61] C. Yang, J. Li, Q. Ni, A. Anpalagan and M. Guizani, "Interference-Aware Energy Efficiency Maximization in 5G Ultra-Dense Networks," in IEEE Transactions on Communications, vol. 65, no. 2, pp. 728-739, Feb. 2017.

[62] D. Zhai, M. Sheng, X. Wang, Z. Sun, C. Xu and J. Li, "Energy-Saving Resource Management for D2D and Cellular Coexisting Networks Enhanced by Hybrid Multiple Access Technologies," in IEEE Transactions on Wireless Communications, vol. 16, no. 4, pp. 2678-2692, April 2017.

[63] W. Ejaz and M. Ibnkahla, "Multiband Spectrum Sensing and Resource Allocation for IoT in Cognitive 5G Networks," in IEEE Internet of Things Journal, vol. 5, no. 1, pp. 150-163, Feb. 2018.

[64] X. Liu, Y. Wang, S. Liu and J. Meng, "Spectrum Resource Optimization for NOMA-Based Cognitive Radio in 5G Communications," in IEEE Access, vol. 6, pp. 24904-24911, 2018.

[65] H. Hosseini, A. Anpalagan, K. Raahemifar and S. Erkucuk, "Wavelet-based cognitive SCMA system for mmWave 5G communication networks," in IET Communications, vol. 11, no. 6, pp. 831-836, 2042017.

[66] X. Liu, M. Jia, Z. Na, W. Lu and F. Li, "Multi-Modal Cooperative Spectrum Sensing Based on Dempster-Shafer Fusion in 5G-Based Cognitive Radio," in IEEE Access, vol. 6, pp. 199-208, 2018.

[67] Z. Song, Q. Ni and X. Sun, "Spectrum and Energy Efficient Resource Allocation WithQoS Requirements for Hybrid MC-NOMA 5G Systems," in IEEE Access, vol. 6, pp. 37055-37069, 2018.

\section{BIOGRAPHY OF AUTHORS}

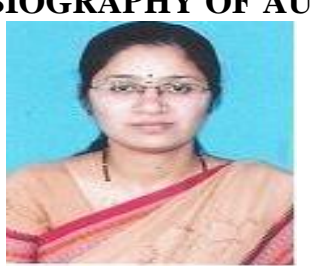

Mrs. Vani B P Completed B.E in E\&C (2005) at Alpha College of Engg. VTU and M.Tech in Digital Electronics (2011) at SSIT, Tumkur, VTU and doing her research in 5G and cognitive networks under VTU. Overall Teaching experience is 12 years.Area of research includes wireless communication and Networking. Presented papers in National/International conferences and Guided UG projects.

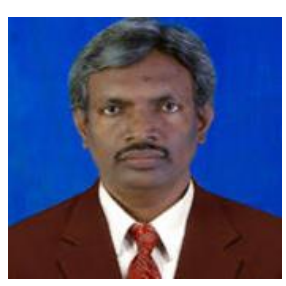

Dr. R Sundaraguru received AMIE inE\&C in 1993, M.E in Communication systems in 1997 at Thiagarajar College of Engg. Madurai and Ph.D. in wireless communication from Anna University Chennai. He is currently working as professor and Head in the department of ECE, Sir MVIT. He has around 3 years of industrial experience as a service/assistant engineer and around 21 years in teaching. His area of research includes wireless communication, Image processing, Networking, VLSI and Embedded systems. To his credit he has published a paper in reputed International journals and IEEE Conferences, about 7 papers in International Journal and 15 paper in National and International conferences. He has coordinated number of Faculty development programme. Guided more than $75 \mathrm{UG}$ and $30 \mathrm{PG}$ projects. He is a member of different professional bodies like Institute of Engineers,Member of IETE and ISTE 